\title{
CALIDAD DE VIDA EN MUJERES CON CÁNCER CÉRVICO-UTERINO, CARTAGENA (COLOMBIA),
} 2012

\author{
Quality of life in women with cervical cancer, \\ Cartagena (Colombia), 2012 \\ Irma Yolanda Castillo-Ávila ${ }^{1}$, Johanna Canencia-Padilla², \\ Mayerlis Zurbarán-Lamadrid², Daniela Pertuz-Cantillo², Marlon Montes-Padilla², \\ Elizabeth Jiménez-Chacón ${ }^{2}$
}

Recibido: mayo 8/14 - Aceptado: febrero 6/15

\section{RESUMEN}

Objetivo: determinar la calidad de vida en mujeres con diagnóstico de cáncer cérvico-uterino de la ciudad de Cartagena (Colombia).

Materiales y métodos: estudio descriptivo, de corte transversal. Se incluyeron mujeres con diagnóstico de cáncer cérvico-uterino en tratamiento que acudieron a una institución pública de salud de primer nivel de complejidad, de la ciudad de Cartagena; fueron excluidas las mujeres que, además del diagnóstico, tenían otra patología o estaban en condiciones críticas. Tamaño muestral 106 mujeres. Muestreo por conveniencia. Se utilizaron dos cuestionarios de la Organización Europea para la Investigación y el Tratamiento del Cáncer (EORTC): el QLQ-C30 para medir calidad de vida en pacientes con cáncer y el QOL-CX24, que es un cuestionario complementario y específico para medir calidad de vida en mujeres con cáncer cérvico-uterino. Se midieron edad, procedencia, estado civil, número de hijos, ocupación, nivel socioeconómico, escolaridad y la calidad de vida según los dos cuestionarios. Se realiza análisis descriptivo.

Enfermera, magíster en Salud Pública, Universidad de Cartagena, Campus de Salud Zaragocilla, Cartagena (Colombia). icastilloa@unicartagena.edu.co 2 Enfermera(o), Universidad de Cartagena, Cartagena (Colombia).
Resultados: participaron 106 mujeres con promedio de edad de 41,3 años (DE $\pm 12,5$ años). Todas las mujeres pertenecen a los estratos socioeconómi$\cos 1$ a 3; el mayor impacto negativo de la enfermedad, según el QLQ-30, se observa en las mujeres de estrato 1. Los aspectos más afectados de la calidad de vida son los relacionados con sus actividades sociales (51,9\%), los estados funcional y físico, la interferencia del tratamiento en su vida familiar (48,1\%), y los problemas económicos causados por la enfermedad (47,2 \%). En la función psicológica, el 87,7\% de las mujeres se han sentido preocupadas por su enfermedad. Para la escala específica QOLCX-24, se encontró que en la dimensión sexual, el $70,7 \%$ ha tenido molestias o irritación vaginal que interfieren en su desempeño.

Conclusión: el cáncer de cuello uterino afecta de manera importante la calidad de vida de las pacientes que lo padecen.

Palabras clave: calidad de vida, indicadores de calidad de vida, cáncer de cuello uterino, salud de la mujer.

\section{ABSTRACT}

Objective: To determine quality of life in women diagnosed with cervical cancer in Cartagena, Colombia. 
Materials and methods: Descriptive, crosssectional study of women with a diagnosis of cervical cancer who were receiving treatment and who came to a public, level I healthcare institution in the city of Cartagena; women suffering with comorbidities or critically ill were excluded. The sample included 106 women, and sampling was done on the basis convenience. Two questionnaires of the European Organization for Research and Treatment of Cancer (EORTC) were used: QLQ-C30 for the measurement of quality of life in cancer patients, and QOL-CX24 as a specific supplementary tool to measure quality of life in patients with cervical cancer. The variables measured were age, place of origin, marital status, number of children, occupation, income bracket, schooling, and quality of life according to the two questionnaires. A descriptive analysis was performed.

Results: Overall, 106 women were included with a mean age of 41.3 years ( $\mathrm{SD} \pm 12.5$ years). All women were of income brackets 1 to 3 , and the greatest impact of the disease was found in income bracket 1, as shown in the QLQ-30. The most affected domains in quality of life were those related to social activites (51.9\%), physical and functional status, and interference of treatment with family life $(48.1 \%)$, while financial issues related to the disease were reported by $47.2 \%$. In terms of psychological function, $87.7 \%$ of the women have been worried by their disease. In the sexual domain under the specific QOL-CX-24 questionnaire, it was found that $70.7 \%$ have issues with vaginal discomfort and irritation, interfering with their sexual performance.

Conclusion: Cervical cancer has a significant impact on the quality of life of the women who suffer from this disease.

Key words: Quality of life, quality of life indicators, cervical cancer, women's health.

\section{INTRODUCCIÓN}

El término calidad de vida (CV) engloba un concepto amplio, que surge a partir de la necesidad de dar respuesta a la valoración objetiva e integral de la salud de las personas (1). El padecimiento de enfermedades crónicas como el cáncer, tiende a desmejorar de manera irreversible la calidad de vida, especialmente porque es inevitable que aparezcan molestias en general y efectos adversos asociados al tratamiento (2).

La Organización Mundial de la Salud (OMS) calcula que cada año se diagnostican a nivel mundial unos 530.000 casos nuevos de cáncer cérvicouterino que requieren tratamiento (3); en cuanto al impacto, la OMS considera que en América el cáncer cérvico-uterino causa la pérdida de 471.000 años de vida ajustados por discapacidad (4). En Colombia, este padecimiento se ubica como el segundo en incidencia y en primer lugar en mortalidad, especialmente en mujeres de bajo nivel socioeconómico, con vida sexual activa y alta multiparidad $(5,6)$. En Cartagena, para el año 2013, se diagnosticaron más de 4.500 mujeres con la enfermedad y es la segunda patología tumoral en importancia en la ciudad $(7,8)$.

La enfermedad genera un impacto negativo en la calidad de vida, alterando las dimensiones psicológica, física, social, espiritual y sexual (9). Además, esta patología y su tratamiento implican una variedad de síntomas, como dolor, náuseas y vómitos, y fatiga, que para la mayoría de las mujeres resultan desagradables (10). Algunos autores han descrito mayor preocupación por la enfermedad entre estas mujeres al término del tratamiento, e incluso tras dos años después de este. En el aspecto emocional, reportan elevados índices de depresión y preocupación por el futuro, acompañados del sentimiento recurrente de miedo a la recidiva $(11,12)$. Asimismo, se han informado importantes alteraciones en el funcionamiento sexual, que generan mayor impacto en el ámbito psicosocial $(9,13)$.

La Organización Europea para la Investigación y el Tratamiento del Cáncer (EORTC), ha diseñado diversas herramientas para la medición de la calidad de vida entre las personas que padecen algún tipo de cáncer, que han sido traducidas, adaptadas y validadas al español (14). Actualmente, el QLQ-C30 
versión 3.0 es el más reciente, y es el instrumento que se utiliza para todos los nuevos estudios sobre la calidad de vida en pacientes con cáncer. Se complementa con módulos específicos de la enfermedad, por ejemplo, cáncer de mama, de pulmón, cabeza y cuello, esófago, ovario, cáncer gástrico, cérvicouterino, el mieloma múltiple, esófago-gástrico, próstata, colorrectal, metástasis hepáticas y cáncer de cerebro (15).

Para el área de la salud, el estudio de la calidad de vida se ha convertido en una herramienta que permite conocer la percepción que tiene el paciente sobre los efectos de una enfermedad, especialmente de las consecuencias sobre su bienestar físico, emocional y social, posibilitando así intervenciones enfocadas en la mejora de la calidad de vida del paciente (16). En Cartagena no se han realizado estudios que den cuenta de la medición de la calidad de vida en las mujeres diagnosticadas con cáncer de cuello uterino, a pesar de la incidencia cada vez mayor de esta enfermedad en la ciudad. Por tanto, el objetivo de este estudio es determinar la calidad de vida en mujeres con diagnóstico de cáncer cérvico-uterino atendidas en una institución de salud de Cartagena, que nos permitan diseñar en el futuro estrategias para reducir su impacto en estas pacientes.

\section{MATERIALES Y MÉTODOS}

Se realizó un estudio descriptivo transversal. Se incluyeron mujeres con diagnóstico de cáncer cérvicouterino, que estaban en una buena condición clínica y recibían tratamiento oncológico en una institución pública de salud de primer nivel de complejidad de la ciudad de Cartagena. Fueron excluidas las mujeres que además del diagnóstico tenían otra patología y se encontraban en condiciones críticas. En esta institución funciona un grupo de atención integral a las mujeres con diagnóstico de cáncer de cuello uterino, organizado por mujeres de la comunidad.

Tamaño de muestra y muestreo. Con una población de referencia de 319 mujeres con cáncer cérvicouterino, se estimó un tamaño de muestra de 106 mujeres, teniendo en cuenta los siguientes parámetros: nivel de confianza del $95 \%$, margen de error del $5 \%$ y proporción esperada de mala calidad de vida del $50 \%$. Fueron seleccionadas mediante un muestreo no probabilístico, utilizando la técnica de bola de nieve. Las mujeres fueron abordadas al salir de la institucion de salud, y se realizaron visitas domiciliarias por estudiantes de ultimo año previamente entrenados.

Para la recolección de información se utilizaron tres instrumentos: una ficha sociodemográfica, y los cuestionarios de la Organización Europea para la Investigación y el Tratamiento del Cáncer (EORTC): el QLQ-C30 versión 3 (15), y el instrumento QOL CX24 específico para cáncer de cuello (17). No se incluyeron los datos clínicos y de tratamiento, porque la encuesta fue autoadministrada y no se tuvo acceso a las historias clínicas.

El instrumento QLQ-C30 (versión 3) consta de tres escalas: escala funcional, que evalúa el funcionamiento físico, emocional, cognitivo, social y de actividades cotidianas; la escala de síntomas, que evalúa la presencia de vómito, náuseas y dolor, entre otros, y la escala que mide el estatus de salud global. Tiene 30 preguntas y se asignan valores entre 1 y 4 (1: en absoluto, 2: un poco, 3: bastante, 4: mucho). Solo los ítems 29 y 30, que conforman la escala de estatus de salud global, se evalúan con puntaje de 1 a 7 (1: pésima, 2: muy mala, 3: mala, 4: regular, 5: buena, 6: muy buena, 7: excelente). Las puntaciones generales del instrumento se estandarizan y se obtiene un puntaje entre 0 y 100 , que determina el nivel de impacto del cáncer en el paciente, a mayor puntaje general mayor impacto de la enfermedad en la calidad de vida (18). Para este estudio, se considerara un impacto negativo de la enfermedad en la calidad de vida, a partir de 60 puntos.

Adicionalmente, se usó el instrumento QOL CX24, que consta de 24 preguntas y es el cuestionario complementario específico para la evaluación de la calidad de vida en la población con diagnóstico de cáncer cérvico-uterino. Consiste en 3 escalas multí́tem y 5 escalas de ítem sencillo. 
Evalúa experiencia de síntomas, imagen corporal y funcionamiento sexual/vaginal (17). Se estandariza en puntajes de 0 a 100 y se consideran puntajes negativos los mayores de 60. Ambos instrumentos han sido utilizados previamente en español en América Latina por Torres et al. (19).

Se midieron variables sociodemográficas como: edad, procedencia, estado civil, número de hijos, ocupación, nivel socioeconómico, escolaridad y calidad de vida según los dos cuestionarios. El nivel socioeconómico en Colombia se clasifica por estratos de 1 a 6 , donde el estrato 1 corresponde al nivel socioeconómico bajo-bajo, el estrato 2 al nivel bajo, el 3 al nivel medio-bajo, el 4 al nivel medio, el 5 al nivel medio-alto y el 6 al nivel alto (20).

Después de recolectar la información, y previa verificación de la calidad de la misma, los datos se almacenaron en una hoja de cálculo del programa Excel, para ser analizados con el software estadístico SPSSS, aplicando un análisis descriptivo.

Las variables continuas se resumieron en medidas de tendencia central y dispersión, y las nominales u ordinales como proporciones.

El estudio fue aprobado por el comité de ética de la institución donde se realizó y, además, se solicitó consentimiento informado por escrito a las participantes, garantizando la confidencialidad de la información obtenida.

\section{RESULTADOS}

Participaron 106 mujeres con una edad promedio de 41,3 años ( $D E=12,5$ años). La mediana de número de hijos fue de 3, el $70 \%$ provenía del área urbana, un 46,2\% vivía en unión libre, el 62,3\% eran amas de casa, el 25,5\% tenía nivel educativo de bachillerato completo; todas provenían de los estratos 1 a 3, y 89,6\% estaban afiliadas al régimen subsidiado por el Estado (tabla 1).

Calidad de vida evaluada por el instrumento (QLQC30). El puntaje global promedio de la escala QLQC30, fue de 57,9; el puntaje promedio más alto o el mayor impacto negativo de la enfermedad sobre la calidad de vida se encontró en las mujeres de estrato socioeconómico 1, con un puntaje de 63,8 $(\mathrm{DE} \pm 14,4)$ (tablas 2 y 3). Respecto a los ítems de la escala funcional, se encontró que 43,4\% de las mujeres manifestaron tener dificultades para hacer actividades que requerían un esfuerzo importante, el $71 \%$ manifestaron requerir estar sentadas o acostadas durante el día. Respecto al funcionamiento emocional o psicológico, 31,2 \% de las mujeres manifestaron sentirse nerviosas, 47,1\% preocupadas y 30,2 \% deprimidas. Para 63,2\% de ellas, su estado físico o el tratamiento médico había interferido considerablemente en su vida familiar. Asimismo, en la escala de síntomas, 49,1\% respondieron

\begin{tabular}{|c|c|c|}
\hline \multicolumn{3}{|c|}{$\begin{array}{c}\text { Tabla } 1 . \\
\text { Distribución según las características } \\
\text { sociodemográficas de las mujeres con cáncer } \\
\text { de cérvix que contestaron la encuesta de calidad } \\
\text { de vida, Cartagena (Colombia), } 2012\end{array}$} \\
\hline Procedencia & $N=106$ & $\%$ \\
\hline Rural & 31 & 29,2 \\
\hline Urbana & 75 & 70,8 \\
\hline \multicolumn{3}{|l|}{ Estado civil } \\
\hline Casada & 25 & 23,6 \\
\hline Separada & 10 & 0,9 \\
\hline Soltera & 19 & 17,9 \\
\hline Unión libre & 49 & 46,2 \\
\hline Viuda & 3 & 2,8 \\
\hline \multicolumn{3}{|l|}{ Ocupación } \\
\hline Ama de casa & 66 & 62,3 \\
\hline Estudia & 5 & 4,7 \\
\hline Trabaja & 35 & 30,2 \\
\hline \multicolumn{3}{|l|}{ Estudios } \\
\hline Analfabeta & 2 & 1,9 \\
\hline Primaria incompleta & 19 & 17,9 \\
\hline Primaria completa & 9 & 8,5 \\
\hline Bachillerato incompleto & 25 & 23,6 \\
\hline Bachillerato completo & 27 & 25,5 \\
\hline Técnico & 21 & 19,8 \\
\hline Universitario & 3 & 2,8 \\
\hline \multicolumn{3}{|l|}{ Afiliación } \\
\hline Contributivo & 11 & 10,4 \\
\hline Subsidiado & 95 & 89,6 \\
\hline
\end{tabular}




\begin{tabular}{|c|c|c|c|c|}
\hline \multicolumn{5}{|c|}{$\begin{array}{c}\text { Tabla 2. } \\
\text { Puntaje global de QLQ-C30 de las mujeres con cáncer de cérvix que contestaron } \\
\text { la encuesta de calidad de vida según estrato, Cartagena (Colombia), } 2012\end{array}$} \\
\hline Estrato & $\mathbf{N}$ & $\%$ & Promedio & Desviación estándar \\
\hline 1 & 52 & 49,1 & 63,8 & 14,4 \\
\hline 2 & 42 & 39,6 & 51,9 & 6,3 \\
\hline 3 & 12 & 11,3 & 53,7 & 8,3 \\
\hline Total & $\mathbf{1 0 6}$ & $\mathbf{1 0 0 , 0}$ & $\mathbf{5 7 , 9}$ & $\mathbf{1 2 , 5}$ \\
\hline
\end{tabular}

\section{Tabla 3.}

Resultados de la calidad de vida en mujeres con cáncer de cérvix medido por el instrumento QLQ-C30, Cartagena (Colombia), 2012

\begin{tabular}{|c|c|c|c|c|c|c|c|c|}
\hline Funcionales & $\begin{array}{c}\text { En } \\
\text { absoluto }\end{array}$ & $\%$ & $\begin{array}{c}\text { Un } \\
\text { poco }\end{array}$ & $\%$ & Bastante & $\%$ & Mucho & $\%$ \\
\hline $\begin{array}{l}\text { Dificultad para hacer actividades que } \\
\text { requieran un esfuerzo importante }\end{array}$ & 60 & 56,6 & 33 & 31,1 & 11 & 10,4 & 2 & 1,9 \\
\hline Dificultad para dar un paseo largo & 55 & 51,9 & 37 & 34,9 & 12 & 11,3 & 2 & 1,9 \\
\hline Dificultad para dar un paseo corto fuera de casa & 75 & 70,8 & 19 & 17,9 & 10 & 9,4 & 2 & 1,9 \\
\hline $\begin{array}{l}\text { ¿Tiene que permanecer en la cama } \\
\text { o sentada en una silla durante el día? }\end{array}$ & 76 & 71,7 & 23 & 21,7 & 6 & 5,7 & 1 & 0,9 \\
\hline $\begin{array}{l}\text { ¿Necesita ayuda para comer, vestirse } \\
\text { o ir al servicio? }\end{array}$ & 88 & 83,0 & 15 & 14,2 & 3 & 2,8 & 0 & 0,0 \\
\hline Impedimento para hacer su trabajo & 63 & 59,4 & 34 & 32,1 & 7 & 6,6 & 2 & 1,9 \\
\hline Impedimento para realizar sus aficiones & 56 & 52,8 & 41 & 38,7 & 6 & 5,7 & 3 & 2,8 \\
\hline $\begin{array}{l}\text { ¿Ha interferido su estado físico o el } \\
\text { tratamiento médico en su vida familiar? }\end{array}$ & 39 & 36,8 & 51 & 48,1 & 14 & 13,2 & 2 & 1,9 \\
\hline $\begin{array}{l}\text { ¿Ha interferido su estado físico o el } \\
\text { tratamiento médico en sus actividades sociales? }\end{array}$ & 37 & 34,9 & 55 & 51,9 & 13 & 12,3 & 1 & 0,9 \\
\hline $\begin{array}{l}\text { ¿Le han causado problemas económicos } \\
\text { su estado físico o el tratamiento médico? }\end{array}$ & 25 & 23,6 & 50 & 47,2 & 26 & 24,5 & 5 & 4,7 \\
\hline Síntomas & $\begin{array}{c}\text { En } \\
\text { absoluto }\end{array}$ & $\%$ & $\begin{array}{c}\text { Un } \\
\text { poco }\end{array}$ & $\%$ & Bastante & $\%$ & Mucho & $\%$ \\
\hline ¿Tuvo asfixia? & 58 & 54,7 & 32 & 30,2 & 15 & 14,2 & 1 & 0,9 \\
\hline ¿Ha tenido dolor? & 12 & 11,3 & 52 & 49,1 & 32 & 30,2 & 6 & 5,7 \\
\hline ¿Necesitó parar para descansar? & 47 & 44,3 & 48 & 45,3 & 9 & 8,5 & 2 & 1,9 \\
\hline ¿Ha tenido dificultades para dormir? & 32 & 30,2 & 49 & 46,2 & 22 & 20,8 & 3 & 2,8 \\
\hline ¿Se ha sentido débil? & 33 & 31,1 & 39 & 36,8 & 25 & 23,6 & 9 & 8,5 \\
\hline ¿Le ha faltado el apetito? & 36 & 34,0 & 39 & 36,8 & 27 & 25,5 & 4 & 3,8 \\
\hline ¿Ha tenido náuseas? & 34 & 32,1 & 50 & 47,2 & 19 & 17,9 & 3 & 2,8 \\
\hline ¿Ha vomitado? & 51 & 48,1 & 51 & 48,1 & 4 & 3,8 & & 0,0 \\
\hline ¿Ha estado estreñida? & 63 & 59,4 & 36 & 34,0 & 6 & 5,7 & 1 & 0,9 \\
\hline ¿Ha tenido diarrea? & 61 & 57,5 & 38 & 35,8 & 6 & 5,7 & 1 & 0,9 \\
\hline ¿Estuvo cansada? & 28 & 26,4 & 51 & 48,1 & 25 & 23,6 & 2 & 1,9 \\
\hline ¿Interfirió algún dolor en sus actividades diarias? & 29 & 27,4 & 58 & 54,7 & 16 & 15,1 & 3 & 2,8 \\
\hline $\begin{array}{l}\text { ¿Ha tenido dificultad de concentrarse en } \\
\text { cosas como leer el periódico, ver la televisión? }\end{array}$ & 73 & 68,9 & 28 & 26,4 & 5 & 4,7 & 0 & 0,0 \\
\hline ¿Se sintió nerviosa? & 37 & 34,9 & 36 & 34,0 & 27 & 25,5 & 6 & 5,7 \\
\hline ¿Se sintió preocupada? & 13 & 12,3 & 43 & 40,6 & 38 & 35,8 & 12 & 11,3 \\
\hline ¿Se sintió irritable? & 42 & 39,6 & 35 & 33,0 & 24 & 22,6 & 5 & 4,7 \\
\hline ¿Se sintió deprimida? & 39 & 36,8 & 35 & 33,0 & 22 & 20,8 & 10 & 9,4 \\
\hline ¿Ha tenido dificultades para recordar cosas? & 77 & 72,6 & 19 & 17,9 & 10 & 9,4 & 0 & 0,0 \\
\hline
\end{tabular}


que el síntoma más frecuente fue el dolor, $67,0 \%$ refirieron dificultad para dormir, 62,3\% falta de apetito y 65,1\% náuseas. En la escala de estatus de salud global, se encontró que para el 35,8 \% de las mujeres su estado de salud durante la semana previa a la aplicación del QLQ-C30 fue regular. La calidad de vida en general durante esta semana también fue percibida como regular en el 29,2\% de las mujeres participantes (tabla 4).

Calidad de vida evaluada por el instrumento QOL-CX24. El promedio general obtenido para el instrumento QOL-CX24, fue de 43. Respecto al funcionamiento sexual, la sensación de acortamiento de la vagina fue el ítem más informado 56,6\%. En la escala de imagen corporal, se encontró que 37,7\% de las encuestadas se sintieron un poco menos atractivas físicamente como consecuencia de su enfermedad, $38,7 \%$ de ellas se sienten un poco menos femeninas, y el 37,7\% manifiesta sentirse un poco desilusionadas con su cuerpo.

Los síntomas urológicos y gastrointestinales más frecuentes fueron: espasmos en el estómago $(41,5 \%)$, incremento ligero de la frecuencia urinaria $(48,1 \%)$ y dolor o escozor al orinar $(50,9 \%)$. Respecto a los síntomas vaginales, $51 \%$ de las mujeres manifestaron que han sentido ligera irritación o molestias vaginales, $33 \%$ han experimentado pérdidas vaginales y $31,1 \%$ refirieren haber experimentado alguna hemorragia vaginal anormal.

En los ítems simples, el 43,4\% ha experimentado hinchazón en piernas, y 47,2 \% ha presentado dolor a nivel lumbar. Al 42,5\% le ha preocupado un poco que el sexo pudiera resultar doloroso, y el $41,5 \%$ lleva una vida sexual poco activa (tabla 5).

\section{DISCUSIÓN}

La calidad de vida de las mujeres con cáncer de cuello cérvico-uterino, de los estratos medio y bajo de la ciudad de Cartagena, según las dos escalas usadas, es impactada de manera negativa por la enfermedad. En general, durante la semana previa a la aplicación del QLQ-C30, la percepción que las mujeres tienen de su propia salud y calidad de vida es regular. Se observa una mayor percepción negativa de la calidad de vida en las mujeres de menor estrato socioeconómico, por lo que podría estudiarse esta relación en investigaciones posteriores.

Nuestros resultados con el instrumento QLQC30, no concuerdan con los resultados encontrados en Cali en el estudio publicado por Parra et al. (9), donde las mujeres con cáncer cérvico-uterino tenían una percepción más negativa de su calidad de vida en general. Estas diferencias pueden derivarse de las diversas condiciones de las mujeres y del apoyo social, familiar, económico y sociosanitario, así como de las diferencias culturales $(10,13,21)$.

Una de las dimensiones de la calidad de vida más afectadas en las mujeres con cáncer de cérvix, es la dimensión psicológica o el funcionamiento emocional. Así lo destacan en Cali Parra y Carrillo, quienes observaron mayor percepción negativa en esta dimensión y mayor presencia de síntomas como ansiedad, depresión, temor a futuros exámenes diagnósticos, temor a cambios en la apariencia, a un segundo cáncer y a manejar la enfermedad en general (9). Torres et al., en Chile (19), muestran que las pacientes estaban emocionalmente más afectadas en relación con las otras dimensiones.

Asimismo, durante el transcurso de la enfermedad y el tratamiento, la percepción de la imagen corporal suele alterarse, lo que expone a las mujeres a sentirse un poco menos atractivas. De igual forma, otros autores $(9,22,23)$ han descrito que en mujeres con diagnóstico de cáncer de cérvix, los cambios de apariencia, la pérdida de peso y la evidencia de los efectos de tratamientos como la radioterapia, significan un factor importante para la percepción negativa de su calidad de vida en cuanto a la dimensión psicológica, y las predisponen a una percepción más negativa de su belleza y feminidad (10).

Un aspecto fundamental para estas mujeres es el disfrute de la sexualidad; al respecto, nuestros datos son similares a lo reportado por Torres et al. en Chile, quienes refieren puntajes altos de preocupación por dolor durante el sexo, aunque en su mayoría manifestaron disfrutar su vida sexual (19). 


\begin{tabular}{|c|c|c|c|c|c|c|c|c|c|c|c|c|c|c|}
\hline \multicolumn{15}{|c|}{ Tabla 4.} \\
\hline Estatus de salud & \multicolumn{2}{|c|}{$\begin{array}{c}1 \\
\text { Pésimo }\end{array}$} & \multicolumn{2}{|c|}{$\begin{array}{c}2 \\
\text { Muy } \\
\text { mala }\end{array}$} & \multicolumn{2}{|c|}{$\begin{array}{c}3 \\
\text { Mala }\end{array}$} & \multicolumn{2}{|c|}{$\begin{array}{c}4 \\
\text { Regular }\end{array}$} & \multicolumn{2}{|c|}{$\begin{array}{c}5 \\
\text { Buena }\end{array}$} & \multicolumn{2}{|c|}{$\begin{array}{c}6 \\
\text { Muy } \\
\text { buena }\end{array}$} & \multicolumn{2}{|c|}{$\begin{array}{c}7 \\
\text { Excelente }\end{array}$} \\
\hline Durante la semana pasada & $\mathbf{N}$ & $\%$ & $\mathbf{N}$ & $\%$ & $\mathbf{N}$ & $\%$ & $\mathbf{N}$ & $\%$ & $\mathbf{N}$ & $\%$ & $\mathbf{N}$ & $\%$ & $\mathbf{N}$ & $\%$ \\
\hline ¿Salud general? & 2 & 1,9 & 2 & 1,9 & 18 & 17,0 & 38 & 35,8 & 31 & 29,3 & 12 & 11,3 & 3 & 2,8 \\
\hline ¿Calidad de vida en general? & 1 & 0,9 & 3 & 2,8 & 18 & 17,0 & 31 & 29,2 & 36 & 34,0 & 15 & 14,2 & 2 & 1,9 \\
\hline
\end{tabular}

Fuente: datos del estudio.

En contraste, en un estudio realizado en Cali, las mujeres con diagnóstico de cáncer cérvico-uterino identificaron una grave afectación en su sexualidad frente a la enfermedad (9). Asimismo, un grupo de mujeres con el diagnóstico evaluadas en Brasil (13), manifiestaron que durante las últimas cuatro semanas no habían tenido relaciones sexuales, y de estas más del 30\% asumía no darle importancia a este aspecto.

Estas diferencias pueden ser explicadas porque los trastornos de la conducta sexual en pacientes oncológicos, dependen de factores como la localización de la afección, el tratamiento, el momento evolutivo, la progresión y el grado de deterioro por la enfermedad $(22,23)$. Estos factores pueden suponer falta de excitación sexual y de placer, ligadas a problemas físicos derivados de la enfermedad como: disminución de la expansión vaginal y disminución de la lubricación de la vagina, regularmente aunados a causas fisiológicas como menopausia prematura e irradiación pélvica. Debido a esto, las mujeres sufren coitos secos y dolorosos, con presencia de daños en la región vaginal y vulvar después de la relación sexual. Además, la atrofia vaginal es un factor que provoca irritación uretral recurrente y produce dispareunia. Por otra parte, los factores emocionales también pueden reducir la excitación sexual de la mujer. Esto puede explicar por qué las mujeres participantes del estudio refirieron mayor preocupación hacia la actividad sexual (13).

La fortaleza de este tipo de investigaciones está en el hecho de que permiten identificar necesidades de la población afectada por el cáncer de cérvix, que no son regularmente evaluadas, y disponer de información para brindar una atención integral y holística. Las principales limitaciones de esta investigación se derivan del diseño descriptivo, y de las que implica el autorreporte. Otra de las principales debilidades de la investigación es no haber tenido acceso a los datos del estadio clínico y tratamiento de las participantes, lo que hubiera permitido un análisis más profundo del impacto de la enfermedad, la severidad y el tratamiento de la misma sobre la calidad de vida de las mujeres con cáncer de cérvix que participaron de esta investigación.

\section{CONCLUSIÓN}

Las mujeres con cáncer de cuello uterino participantes experimentan en la evaluación general un impacto negativo de la enfermedad en su calidad de vida; según su percepción, en la semana previa a la aplicación del QLQ-C30 calificaron de manera regular su estatus de salud global y su calidad de vida.

La funcionalidad emocional o la dimensión psicológica es la más afectada en las mujeres participantes, debido a la constante preocupación por su salud y a los sentimientos de tristeza e irritabilidad que experimentan. De igual forma, la dimensión sexual se ve afectada por síntomas vaginales como irritación, pérdidas vaginales y disminución de la actividad sexual; las mujeres refieren alteraciones relacionadas con la imagen corporal, manifestando sentirse menos atractivas físicamente. 


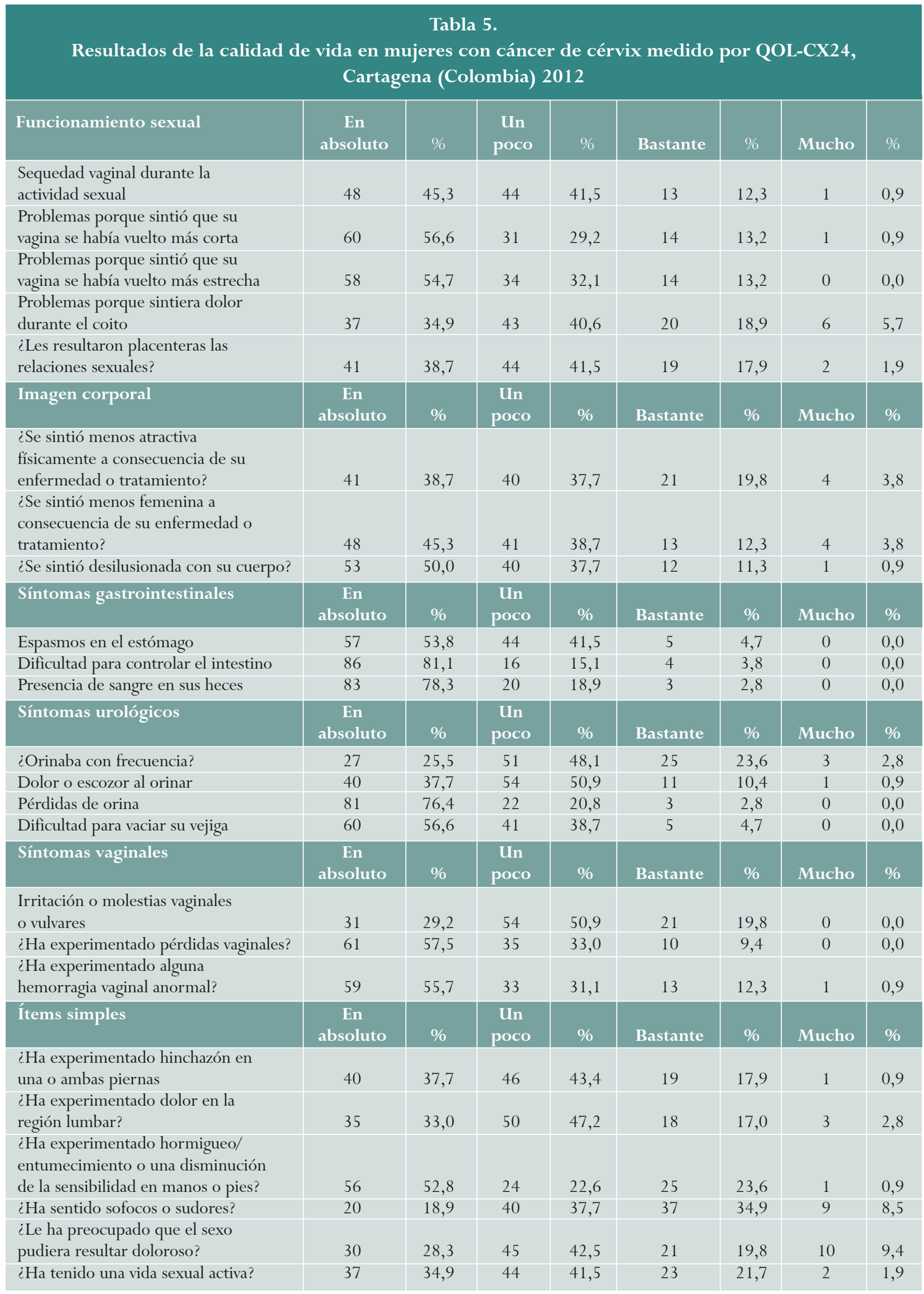

Fuente: datos del estudio. 


\section{REFERENCIAS}

1. Schwartzmann L. Calidad de vida relacionada con la salud: aspectos conceptuales. Cienc enferm. 2003;9:9-21.

2. Etxeberría Y, Pérez J, Badiola C, Quintana J, Padierna A, Aróstegui I et al. Evaluación de la calidad de vida en pacientes con cáncer de pulmón. Gac Sanit. 2001; $15: 25-31$.

3. Organización Panamericana de la Salud. Nota de orientación de la OPS/OMS: Prevención y control integrales del cáncer cérvico-uterino: un futuro más saludable para niñas y mujeres. Washington, D.C.: OPS; 2013. [Visitado 2014 Feb 23]. Disponible en: http://www.paho.org/hq/index.php?option $=\mathrm{com}_{-}$ docman\&task $=$ doc_view\&gid $=22013 \&$ Itemid

4. Lewis M. Análisis de la situación del cáncer cérvicouterino en América Latina y el Caribe. Organización Panamericana de la Salud; 2004. [Visitado 2014 Ene 16]. Disponible en: http:/www.paho.org/Spanish/AD/ DPC/NC/pcc-cc-sit-lac.pdf

5. Ministerio de Salud y Protección Social. 1er Informe Nacional de Calidad de la Atención en Salud "INCAS Colombia 2009”, por el cual se establece el Sistema de información para la Calidad. Bogotá: Ministerio de Salud y Protección social; 2009. [Visitado 2013 Dic 15]. Disponible en: http://calisaludable.cali.gov. co/secretario/2010_incas/MPS_INCAS_FINAL.pdf

6. Liga contra el Cáncer Seccional Bogotá. Tipos de cáncer (Cáncer Cérvico-Uterino). [Visitado 2013 Feb 20]. Disponible en: http://www.ligacontraelcancer. com.co/cancer-de-cuello-uterino/factores-de-riesgo

7. Redondo C, Estrada H, Herrera S, Revueltas W. Hospital Universitario del Caribe. Registro poblacional del cáncer en Cartagena de Indias. Año 2003. [Visitado 2013 Feb 23]. Disponible en: http://www.hucaribe. gov.co/novedades/registro_poblacional_cancer.pdf.

8. Mapa cancerológico de la ciudad de Cartagena. Periódico digital de la Universidad de Cartagena, Universo U. [Visitado 2014 Feb 2]. Disponible en: http://periodicouniversou.unicartagena.edu.co/index. php/noticias/item/307-mapa-cancerologico-en-laciudad-de-cartagena.
9. Parra L, Carrillo G. Calidad de vida de mujeres en situación de enfermedad crónica de cáncer de cérvix. Revista Avances en Enfermería. 2011;29:87-96.

10. Montes L, Mullins P, Urrutia M. Calidad de vida en mujeres con cáncer cérvico-uterino. Revista Chilena de Obstetricia y Ginecología. 2006;71:129-234.

11. Wenzel L, DeAlba I, Habbal R, Kluhsman BC, Fairclough D, Krebs LU, et al. Quality of life in long-term cervical cancer survivors. Gynecol Oncol. 2005;97: 310-7.

12. Klee M, Thranov I, Machin D. Life after radiotherapy: the psychological and social effects experienced by women treated for advanced stages of cervical cancer. Gynecol Oncol. 2000;76:5-13.

13. Cassemiro W, Kimura M. Calidad de vida relacionada a la salud de mujeres con cáncer de cuello uterino. Rev Latino-Am Enfer. 2010;18:65-72.

14. Arraras J, Martínez M, Manterota A. La evaluación de la calidad de vida del paciente oncológico. El grupo de calidad de vida de la EORTC. Psico-oncología. 2004;1:87-98.

15. Aaronson NK, Ahmedzai S, Bergman B, Bullinger M, Cull A, Duez NJ, et al. The European Organization for Research and Treatment of Cancer QLQ-C30: a quality-of-life instrument for use in international clinical trials in oncology. J Natl Cancer Inst. 1993;85:365-76.

16. Cerda P, Pino P, Urrutia MT. Calidad de vida en mujeres histerectomizadas. Rev Chil Obstel Ginecol. 2006;71:216-21.

17. Greimel ER, Kuljanic Vlasic K, Waldenstrom AC, Duric VM, Jensen PT, Singer S, et al. The European Organization for Research and Treatment of Cancer (EORTC). Quality-of-Life questionnaire cervical cancer module: EORTC QLQ-CX24. Cancer. 2006; 107:1812-22

18. Fayers P, Aaronson N, Bjordal K, Groenvold M, Curran D, Bottomley A. The EORTC QLQ-C30 Scoring Manual (3rd. edition). Brussels: European Organization for Research and Treatment of Cancer; 2001. 
19. Torres P, Irarrázaval O. Fasce G, Urrejola R, Pierotic M, León $\mathrm{H}$ et al. Calidad de vida en pacientes con cáncer de cuello uterino: experiencia FALP. Revista Chilena de Obstetricia y Ginecología. 2010;75:383-9.

20. Departamento Nacional de Estadística Colombia (DANE). Nivel Socioeconómico preguntas frecuentes. [Visitado 2014 Nov 20]. Disponible en: http:// www.dane.gov.co/index.php/estratificacionsocioeconomica/preguntas-frecuentes

21. Vinaccia S, Orozco L. Aspectos psicosociales asociados con la calidad de vida de personas con enfermedades crónicas. Diversitas. 2005;1:125-37.
22. Molina S, Aguilar B, Asencio G, Martin M, Sánchez F. Valoración de la información recibida por mujeres con cáncer de cérvix en remisión tras tratamiento con radioterapia. Nure Investigación. 2006;24:1-19.

23. De Groot J, Mah K, Fyles A, Winton S, Greenwood S, Depetrillos A, et al. The psychosocial impact of cervical cancer among affected women and their partners. Int J Gynecol Cancer. 2005;15:918-25. 